\title{
Contextes de production et LAE en français : caractéristiques pragmatiques et syntaxiques
}

\author{
Sophie Kern \\ Dynamique Du Langage (DDL) \\ sophie.kern@univ-lyon2.fr \\ Florence Chenu \\ Dynamique Du Langage (DDL) \\ Florence.Chenu@univ-lyon2.fr
}

\section{Introduction}

Les recherches translinguistiques sur le développement du lexique chez l'enfant dégagent des similitudes dans la trajectoire et la composition du vocabulaire durant la deuxième année de vie de l'enfant. Dans un éventail relativement large de langues, le vocabulaire des enfants présente un biais nominal au cours de la période, suivi d'une augmentation des prédicats et des mots fonctionnels. Une première explication de nature conceptuelle est abandonnée au profit d'une explication linguistique en raison de tendances contraires observées dans le vocabulaire d'enfants en voie d'acquisition de langues asiatiques : le biais nominal ou verbal serait à mettre en relation avec le type d'input que l'enfant reçoit à travers le langage adressé à l'enfant (LAE). Une explication impliquant l'influence du type d'activité sur la composition du vocabulaire vient compléter ce tableau : seul ce type d'explication permet de rendre compte de résultats divergents obtenus au sein d'une même langue.

Dans ce travail, nous cherchons à expliquer le biais nominal observé chez les enfants français au début du développement lexical. Nous nous intéressons en particulier aux caractéristiques pragmatiques et syntaxiques de l'input maternel qui peuvent favoriser ce biais nominal. Notre objectif est de vérifier si ces caractéristiques changent en fonction de la situation ou si elles restent les mêmes à travers différents contextes de production.

\section{2 États des lieux}

La domination des noms sur les autres catégories grammaticales pendant la période des premiers mots est observée dans l'étude translinguistique princeps de Gentner (1982) chez des enfants apprenant l'anglais, l'allemand, le japonais, le turc, le kaluli et le chinois mandarin. Des résultats semblables sont répliqués en anglais (voir entre autres, Bornstein et al., 2004 ; Fenson et al., 1994 ; Goldfield, 2000) mais également en italien (Bornstein et al., 2004 ; Caselli et al., 1995 ; Tardif et al., 1997), hébreu (Bornstein et al., 2004 ; Dromi, 1987 ; Maital et al., 2000), espagnol (Jackson-maldonado et al., 1993) et en flamand (Bornstein et al., 2004 ; De Houwer \& Gillis, 1998; Gillis \& Verlinden, 1988). Dans cet ensemble de langues, les enfants passent de la référence avec un nombre plus important de noms dans leur répertoire à la prédication (verbes et adjectifs) pour finalement entrer dans la grammaire par le biais d'outils grammaticaux toujours plus nombreux et variés. Une même trajectoire est identifiée en français de France (Bassano, 2000 ; Kern, 2007 ; Kern \& Gayraud, 2010 ; Parisse \& Le Normand, 2000) et en français canadien (Poulain-Dubois et al., 1995) et ce à la fois sur la base de données spontanées ou quasiexpérimentales et de comptes-rendus parentaux. Parisse et Le Normand (2000) enregistrent 27 enfants français âgés de 2 ans en France lors de jeux symboliques. Ils rapportent un plus grand nombre de types de noms que de verbes : $29 \%$ des noms sont produits dans des énoncés à un seul mot et $44 \%$ dans des énoncés plus longs contre seulement 18 et $22 \%$ des verbes. En se basant à la fois sur une étude longitudinale d'un enfant français entre 14 et 30 mois et une étude transversale de 12 enfants de 20 mois et 12 de 30 mois, Bassano (1998) et Bassano et al. (1998) font apparaître que les noms et les items paralexicaux (interjections, particules...) sont les plus fréquents jusqu'à 20 mois pour ensuite laisser place 
aux prédicats et mots grammaticaux. Les résultats obtenus à partir de l'Inventaire Français du Développement Communicatif (Kern, 2007 ; Kern \& Gayraud, 2010) vont dans le même sens : entre 8 et 16 mois, les noms représentent $80 \%$ des mots produits; de 16 à 30 mois, la catégorie des noms est toujours la catégorie dominante $(58,5 \%)$ même si le pourcentage est plus faible que chez les enfants plus jeunes en raison d'une augmentation significative des verbes et des adjectifs.

Gentner (1982) propose une explication conceptuelle à cette trajectoire particulière. Elle avance «the Natural Partitions Hypothesis » hypothèse selon laquelle les noms seraient acquis avant les prédicats (verbes et adjectifs) car leurs référents sont plus accessibles et plus stables que ceux des prédicats. Cette hypothèse est progressivement abandonnée avec la mise en évidence de trajectoires acquisitionelles différentes chez des apprenants de chinois mandarin (Tardif, 1996), de coréen (Choi \& Gopnik, 1995; Gopnik et al., 1996; Cheng, 1994) et de tzotzil (de Leon, 1998). Dans ces études, le biais dans la composition du vocabulaire porte sur le verbe et non pas sur le nom. Tardif (1996) par exemple, rapporte que 9 enfants chinois sur 10, âgés de 22 mois produisent significativement plus de types de verbes différents que de noms communs. Les auteurs concluent à un rôle prédominant du langage adressé à l'enfant sur les préférences précoces des enfants.

Les différences de fréquence des noms par rapport aux verbes dans le premier lexique des enfants sont expliquées par des caractéristiques particulières de l'input: les unes favorisant la production des noms, les autres celle des verbes. Ces caractéristiques de l'input évoquées dans la littérature (Bornstein et al., 2004 ; Ogura et al., 2006 ; Tardif et al., 1997) sont la fréquence des noms vs verbes, la transparence du système morphologique nominal vs. verbal, la position saillante des noms vs. verbes, la focalisation particulière des mères sur une catégorie grammaticale plutôt qu'une autre. Dans ce travail, seuls les deux derniers points sont considérés, à savoir la position des noms et des verbes dans les énoncés ainsi que l'orientation des énoncés des adultes vers la production chez les enfants d'une classe grammaticale particulière.

La position finale (et dans une moindre mesure la position initiale) sont des positions auxquelles les enfants sont particulièrement attentifs (Slobin, 1985). L'ordre des constituants des phrases varie en fonction des langues : dans certaines ce sont les noms qui sont placés en position finale et donc en position saillante, dans d'autres, ce sont les verbes. En anglais par exemple, compte tenu d'un ordre des mots SVO plutôt stable, une emphase est placée sur les noms. Par ailleurs dans le langage que les parents anglophones adressent à leur enfant, les noms apparaissent plus fréquemment que les verbes dans les énoncés à un seul mot et tendent à être davantage placés en fin des longs énoncés (Goldfield, 1993 ; Tardif et al., 1997). Il en va de même pour les phrases en français où le sujet est obligatoire et dont l'ordre dominant est SVO (Harris, 1990). À l'inverse, certaines propriétés structurales des langues asiatiques favorisent l'emploi des verbes. En chinois mandarin par exemple, les verbes apparaissent plus souvent en fin d'énoncés que les noms. Ce sont donc les caractéristiques syntaxiques des langues comme l'ordre des mots qui rendent certains éléments plus ou moins saillants et qui peuvent expliquer le déséquilibre noms/verbes observé dans les premiers vocabulaires des enfants.

L'aspect pragmatique des énoncés adressés à l'enfant doit aussi être pris en compte pour expliquer le déséquilibre entre noms et verbes au sein des productions enfantines. En effet, plusieurs recherches (Choi \& Gopnik, 1995; Choi, 2000; Kim et al., 2000; Fernald \& Morikawa, 1993; Ogura et al., 2006) observent que les énoncés du locuteur peuvent focaliser l'attention de l'interlocuteur sur une catégorie grammaticale plutôt qu'une autre. Deux principaux types d'énoncés sont définis dans cet ensemble de travaux : les énoncés orientés vers les objets et ceux orientés vers les activités. Les résultats soulignent des différences stylistiques entre les mères qui appartiennent à des cultures linguistiques différentes. Ainsi les mères coréennes produisent plus d'énoncés orientés vers les activités que les mères anglophones (Choi, 2000 ; Choi \& Gopnik, 1995 ; Kim et al., 2000). Mais ces préférences pragmatiques varient également beaucoup en fonction du contexte, contexte pris ici dans le sens de type d'activité dans laquelle les interlocuteurs sont impliqués lors de l'échange. Tardif et al. (1999) montrent que les mères parlant le chinois mandarin posent davantage de questions que les mères anglophones pour éliciter des noms de la part de leurs enfants dans le contexte de lecture partagée. Choi (2000), dans son étude comparative sur 20 dyades mère/enfant coréennes et 20 dyades anglophones trouvent à la fois un effet de la langue mais également 
un effet du contexte: alors que les mères anglophones orientent leurs énoncés sur les noms dans la lecture d'album sans texte et dans les séances de jeux, les mères coréennes ne le font que dans le contexte de la lecture partagée d'album. Dans le contexte du jeu, elles produisent plus de verbes et focalisent l'attention de l'enfant davantage sur les actions.

\section{Questions et hypothèses de recherche}

Cet état de l'art à propos du déséquilibre entre les noms et les verbes dans le vocabulaire des enfants ainsi que les explications que l'on donne de ce déséquilibre nous conduit à nous interroger sur le comportement des mères françaises. Compte-tenu des caractéristiques morpho-syntaxiques de la langue française qui tendent à accorder une place privilégiée aux noms et du biais nominal observé dans les données des enfants français, nous cherchons à vérifier si les mères attirent davantage l'attention de leurs enfants sur les noms que sur les verbes, et si oui, par quels procédés linguistiques? Nous cherchons à savoir si ces procédés se retrouvent dans des situations différentes : l'une favorisant la production de noms (lecture d'album sans texte) et l'autre celle de verbes (jeu avec maison de poupée). Pour ce faire nous déterminons dans un premier temps la cible des énoncés des mères: les énoncés des mères sont-ils orientés majoritairement vers les noms ou plutôt vers les verbes? Dans un deuxième temps, la position des noms et des verbes est étudiée en fonction du contexte de production.

Nous nous attendons en particulier à ce que les mères attirent davantage l'attention de leur enfant sur les objets et personnes et que ce type de focalisation soit présent dans les deux contextes de production. Par ailleurs, nous pensons trouver davantage de noms que de verbes en position saillante, c'est-à-dire, soit en énoncés monomots, soit en position finale dans des énoncés multimots dans les deux contextes de production.

\section{Méthode}

\subsection{Sujets}

20 enfants français âgés de 16 à 34 mois (âge moyen : 23 mois) et leurs mères font partie de l'étude. Toutes les mères ont un niveau d'études allant de bac à bac +3 et travaillent à plein temps. Les familles vivent à Lyon dans un contexte linguistique monolingue. Les enfants (11 filles et 9 garçons) ont un développement physique, intellectuel et social conforme à la norme nationale.

\subsection{Tâches}

Les sujets sont filmés en France au domicile des parents en interaction avec leur mère lors de deux situations différentes: la lecture partagée d'un album sans texte et un jeu avec une maison de poupées. Chaque situation dure 10 minutes. L'album (The Farm Picture Book, Amery \& Cartwright, 1986) utilisé pour éliciter la lecture partagée contient 5 scènes de ferme qui représentent de nombreux personnages impliqués dans des événements variés. Au cours de la session de jeu, les dyades mère-enfant utilisent une maison miniature composée de plusieurs pièces et d'objets mobiles (personnages, frigidaire, télévision, miroir...). Dans les deux situations, les mères ont pour consigne de se comporter le plus naturellement possible.

\subsection{Procédure de transcription et de codage}

Tous les énoncés des mères et des enfants sont transcrits orthographiquement et saisis sous le logiciel CLAN (MacWhinney, 2000) par des locuteurs natifs du français. Un codage pragmatique et syntaxique est réalisé sur la production des mères. 
Pour ce qui est de la pragmatique, chaque énoncé de la mère est catégorisé selon l'intention communicative qu'il vise. Les énoncés visant à concentrer l'attention de l'enfant sur un objet ou une personne de son environnement sont codés comme des énoncés orientés vers les objets (EOO). Ces énoncés prennent la forme d'un étiquetage (c'est une maison), de questions visant l'identification d'objets ou de personnes (qu'est-ce que c'est ça?) ou encore d'énoncés visant à attirer l'attention de l'enfant sur des entités (regarde ce petit poulet). Les énoncés codés comme énoncés orientés vers les actions (EOA) quant à eux ont pour but de renforcer l'attention des enfants sur une action ou une activité. Ce sont des énoncés décrivant des activités (cet enfant est en train de donner quelque chose à manger), des questions sur la nature des actions (qu'est-ce qu'il fait), ou encore des demandes d'action (tu mets ça dans la cuisine). Une catégorie « autres » comprend les énoncés ambigus et les énoncés sans verbe ou sans nom.

Le codage syntaxique consiste à coder la longueur des énoncés de la mère ainsi que la nature grammaticale de chaque mot qui compose ces énoncés. Les marqueurs énonciatifs, les interjections et les onomatopées sont exclus de cette analyse dans la mesure où une étude préliminaire n'a révélé aucun effet de leur présence sur les résultats obtenus. Pour la longueur des énoncés, 6 catégories ont été définies :

1. Nom seul, nom propre ou commun (nom)

2. Verbe lexical seul (verbe)

3. Pronom + verbe lexical (pro+verbe)

4. Déterminant + nom $(\operatorname{det}+$ nom $)$

5. «Autres» seul (autres)

6. Enoncés plus longs que les catégories 1 à 5 .

Les catégories 1 à 5 sont considérées comme comprenant des énoncés monomots alors que la catégorie 6 est celle des énoncés multimots.

Dans un deuxième temps, on observe la nature des mots en position initiale et finale dans les énoncés. Comme notre intérêt porte sur les noms et les verbes, 5 catégories sont utilisées :

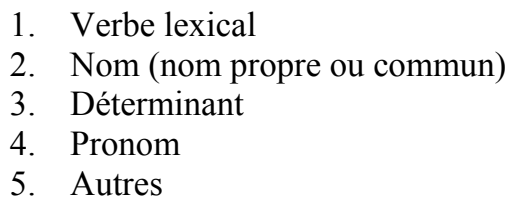

Les catégories 3 et 4 sont importantes dans la mesure où les déterminants en français précèdent toujours un nom et que les pronoms dans la plupart des cas se placent devant un verbe.

\section{Résultats}

Nous présentons dans un premier temps les caractéristiques principales de nos données. Nous présentons ensuite les résultats concernant les intentions communicatives en fonction des deux contextes communicatifs (Lecture d'Album - LA et jeu avec la Maison de Poupée - MP). Nous nous intéressons ensuite à la composition linguistique des énoncés en fonction des contextes communicatifs (CC) puis en fonction des intentions communicatives (IC). Enfin nous présentons les résultats concernant les éléments en position initiale et en position finale en fonction des contextes communicatifs et des intentions communicatives.

\subsection{Résumé des données}

Notre corpus se compose de 38 enregistrements transcrits et codés dont les caractéristiques principales (nombre d'énoncés maternels codés pour chaque enregistrement dans les 2 conditions, totaux et moyennes) sont présentées dans le Tableau 1. 


\begin{tabular}{|c|c|c|}
\hline Enregistrement & $\begin{array}{c}\text { Nombre d'énoncés en } \\
\text { condition LA }\end{array}$ & $\begin{array}{c}\text { Nombre d'énoncés en } \\
\text { condition MP }\end{array}$ \\
\hline MAR & 176 & 225 \\
\hline ALB & 102 & 108 \\
\hline ALE & 181 & 191 \\
\hline BEN & 115 & 170 \\
\hline CLA & 109 & 107 \\
\hline GUI & 92 & 133 \\
\hline HEC & 107 & 128 \\
\hline LUC & 151 & 124 \\
\hline LYL & 143 & 120 \\
\hline NAI & 110 & 117 \\
\hline NIA & 179 & 136 \\
\hline NIN & 237 & 214 \\
\hline NOE & 123 & 121 \\
\hline ORI & 170 & 106 \\
\hline PRU & 172 & 155 \\
\hline RAP & 90 & 84 \\
\hline TIM & 75 & 57 \\
\hline TOM & 174 & 114 \\
\hline ZO2 & 178 & 174 \\
\hline ZOE & 93 & 106 \\
\hline & $\mathbf{2 6 6 9}$ & $\mathbf{2 5 8 0}$ \\
\hline Total & $\mathbf{1 4 0 , 4 7}$ & $\mathbf{1 3 5 , 7 9}$ \\
\hline Moyenne & & \\
\hline
\end{tabular}

Tableau 1. Nombre d'énoncés maternels codés par enregistrement pour chaque condition

Les 2 contextes communicatifs sont comparables en longueur: la différence entre le nombre moyen des énoncés en lecture d'album et celui des énoncés au cours du jeu avec la maison de poupée est non significative $(\mathrm{t}(19)=-0,618 ; \mathrm{p}=0,5442)$

\subsection{Intentions Communicatives (IC) en fonction des deux contextes communicatifs (Lecture d'Album - LA et jeu avec la Maison de Poupée - MP)}

Le Tableau 2 présente le pourcentage moyen (par enregistrement) d'EOO et d'EOA ainsi que l'écart-type. Un $t$ de Student pour séries appariées a été calculé sur les rapports EOO/EOA ( $t(19)=-3,784 ; \mathrm{p}=$ $0,0013)$. Le contexte LA présente en moyenne plus d'EOO que d'EOA, tandis que le contexte MP présente plus d'EOA que d'EOO. 


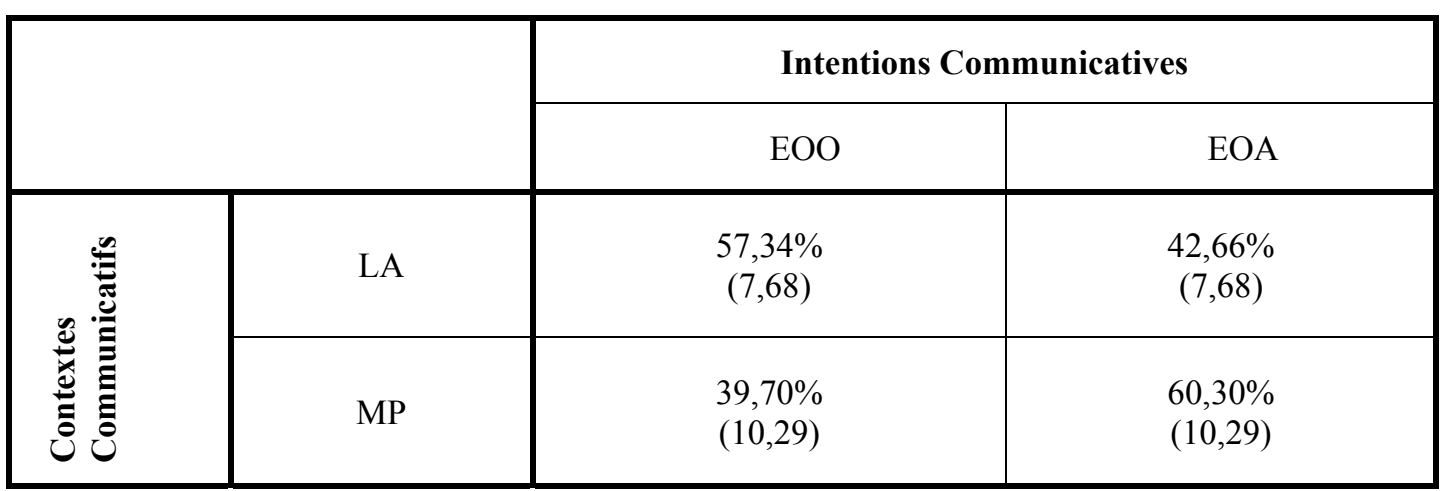

Tableau 2. Pourcentage moyen des énoncés maternels de type EOO et EOA en fonction du contexte communicatif (écart-type entre parenthèses).

\subsection{Composition linguistique des énoncés maternels en fonction des contextes communicatifs (CC)}

\begin{tabular}{|c|c|c|c|c|c|c|c|}
\hline & \multirow{2}{*}{$\begin{array}{c}\begin{array}{c}\text { Énoncés } \\
\text { multimots }\end{array} \\
\text { multimots }\end{array}$} & \multicolumn{5}{|c|}{ Énoncés monomots } \\
\hline & & & $\operatorname{det}+$ nom & pro+verbe & nom & verbe & autres \\
\hline$\stackrel{n}{E}$ & LA & $\begin{array}{c}88,70 \% \\
(5,13)\end{array}$ & $\begin{array}{l}6,31 \% \\
(3,37)\end{array}$ & $\begin{array}{l}2,79 \% \\
(1,92)\end{array}$ & $\begin{array}{l}0,72 \% \\
(1,44)\end{array}$ & $\begin{array}{l}0,51 \% \\
(0,94)\end{array}$ & $\begin{array}{l}0,97 \% \\
(1.52)\end{array}$ \\
\hline Uُ & MP & $\begin{array}{c}90,64 \% \\
(4,55)\end{array}$ & $\begin{array}{l}2.36 \% \\
(2,38)\end{array}$ & $\begin{array}{l}3,34 \% \\
(2,17)\end{array}$ & $\begin{array}{l}0,59 \% \\
(1,04)\end{array}$ & $\begin{array}{l}2,31 \% \\
(3,07)\end{array}$ & $\begin{array}{l}0,75 \% \\
(0,95)\end{array}$ \\
\hline
\end{tabular}

Tableau 3. Pourcentage moyen d'énoncés multimots et monomots en fonction des contextes communicatifs (écart-type entre parenthèses).

Le Tableau 3 présente le pourcentage moyen d'énoncés multimots et monomots en fonction des contextes communicatifs. Les écart-types sont donnés entre parenthèses. Dans les deux contextes communicatifs, les énoncés multimots sont très largement privilégiés. On note que les énoncés monomots comportant un verbe se trouvent davantage en contexte MP que LA $(\mathrm{t}(19)=-3,302 ; \mathrm{p}=0,0037$; calculé sur la racine de l'arc sinus du pourcentage de verbes).

\subsection{Composition linguistique des énoncés maternels en fonction des intentions communicatives (IC)}

Le Tableau 4 présente le pourcentage moyen d'énoncés multimots et monomots en fonction des intentions communicatives. Les écart-types sont donnés entre parenthèses. Dans les deux cas, les énoncés multimots sont très largement privilégiés. On note que les énoncés monomots comportant un verbe se trouvent davantage en EOA que EOO $(\mathrm{t}(19)=-5,337 ; \mathrm{p}<0,001$; calculé sur la racine de l'arc sinus du pourcentage de verbes). 


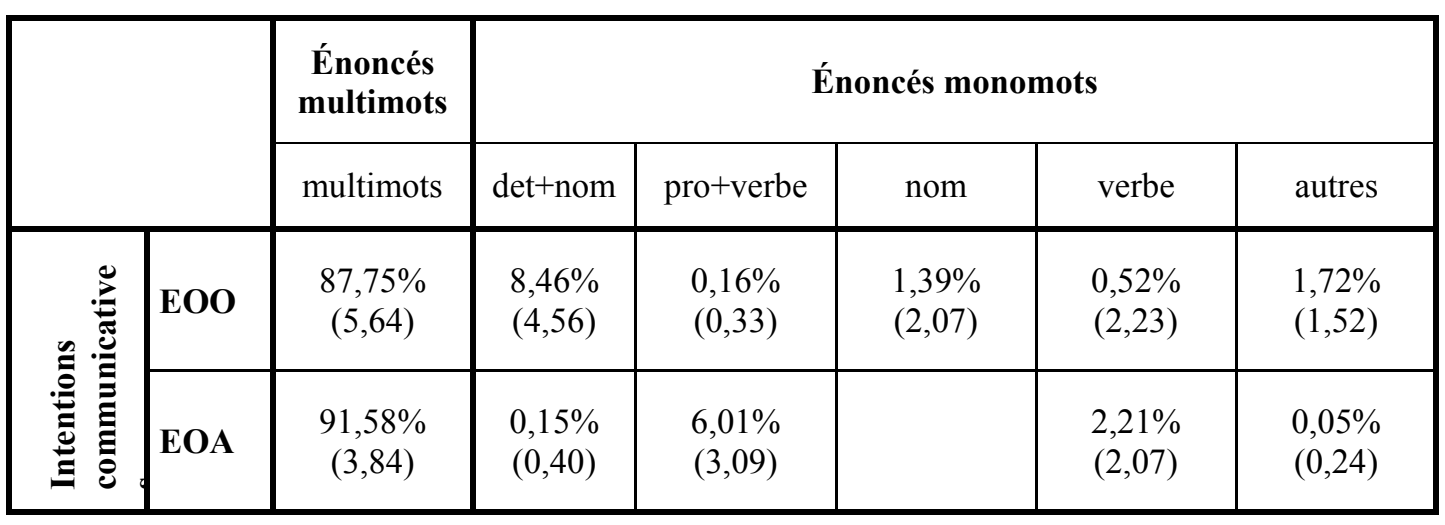

Tableau 4. Pourcentage moyen d'énoncés multimots et monomots en fonction des intentions communicatives (écart-type entre parenthèses).

\section{5 Éléments en position initiale et en position finale en fonction des contextes communicatifs}

Le tableau 5 présente le pourcentage moyen d'énoncés terminés par un nom, un verbe ou un élément d'une autre catégorie en fonction des contextes communicatifs. On note que la proportion de verbes en position finale, c'est-à-dire en position saillante, est plus importante en contexte MP que LA (t(19) = $5.210 ; \mathrm{p}<0.0001 ;$ calculé sur la racine de l'arc sinus du pourcentage de verbes).

\begin{tabular}{|c|c|c|c|}
\hline & Noms & Verbes & Autres \\
\hline LA & $\begin{array}{c}60,49 \% \\
(9,57)\end{array}$ & $\begin{array}{c}13,90 \% \\
(5,02)\end{array}$ & $\begin{array}{c}25,61 \% \\
(6,90)\end{array}$ \\
\hline MP & $\begin{array}{c}4,03 \% \\
(6,34)\end{array}$ & $\begin{array}{c}23,81 \% \\
(7,21)\end{array}$ & $\begin{array}{c}28,16 \% \\
(5,57)\end{array}$ \\
\hline
\end{tabular}

Tableau 5. Pourcentage moyen d'énoncés terminés par un nom, un verbe ou un élément d'une autre catégorie en fonction des contextes communicatifs (écart-type entre parenthèses).

Le Tableau 6 présente le pourcentage moyen d'énoncés initiés par un nom, un verbe, un déterminant, un pronom ou un élément d'une autre catégorie en fonction des contextes communicatifs. Le pourcentage moyen d'énoncés initiés par un verbe tend à être plus élevé en contexte MP que LA mais de façon non significative $(\mathrm{t}(19)=1,683 ; \mathrm{p}=0,1087$; calculé sur la racine de l'arc sinus du pourcentage d'énoncés initiés par un verbe). 


\begin{tabular}{|c|c|c|c|c|c|}
\hline & Noms & Déterminants & Verbes & Pronoms & Autres \\
\hline LA & $\begin{array}{c}1,16 \% \\
(2,41)\end{array}$ & $\begin{array}{c}16,86 \% \\
(3,59)\end{array}$ & $\begin{array}{c}3,67 \% \\
(3,17)\end{array}$ & $\begin{array}{c}44,82 \% \\
(7,48)\end{array}$ & $\begin{array}{c}33,49 \% \\
(7,22)\end{array}$ \\
\hline MP & $\begin{array}{c}2,34 \% \\
(2,85)\end{array}$ & $\begin{array}{c}7,49 \% \\
(3,80)\end{array}$ & $\begin{array}{c}6,50 \% \\
(4,95)\end{array}$ & $\begin{array}{c}54,04 \% \\
(6,79)\end{array}$ & $\begin{array}{c}29,63 \% \\
(8,42)\end{array}$ \\
\hline
\end{tabular}

Tableau 6. Pourcentage moyen d'énoncés initiés par un nom, un verbe, un déterminant, un pronom ou un élément d'une autre catégorie en fonction des contextes communicatifs (écart-type entre parenthèses).

\section{6 Éléments en position initiale et en position finale en fonction des intentions communicatives}

Le tableau 7 présente le pourcentage moyen d'énoncés terminés par un nom, un verbe ou un élément d'une autre catégorie en fonction des intentions communicatives. On note que le pourcentage moyen de verbes en EOA est significativement plus élevé qu'en EOO $(t(19)=-16,631 ; \mathrm{p}<0,0001$; calculé sur la racine de l'arc sinus du pourcentage de verbes)

\begin{tabular}{|c|c|c|c|}
\hline & Noms & Verbes & Autres \\
\hline \multirow{2}{*}{ EOO } & $\begin{array}{c}63,64 \% \\
(7,93)\end{array}$ & $\begin{array}{c}4,85 \% \\
(3,50)\end{array}$ & $\begin{array}{c}31,50 \% \\
(6,77)\end{array}$ \\
\hline \multirow{2}{*}{ EOA } & $\begin{array}{c}4,20 \% \\
(7,45)\end{array}$ & $\begin{array}{c}32,31 \% \\
(7,06)\end{array}$ & $\begin{array}{c}22,49 \% \\
(5,43)\end{array}$ \\
\hline
\end{tabular}

Tableau 7. Pourcentage moyen d'énoncés terminés par un nom, un verbe ou un élément d'une autre catégorie en fonction des intentions communicatives (écart-type entre parenthèses).

Le Tableau 8 présente le pourcentage moyen d'énoncés initiés par un nom, un verbe, un déterminant, un pronom ou un élément d'une autre catégorie en fonction des intentions communicatives. Le pourcentage moyen d'énoncés initiés par un verbe est significativement plus élevé en contexte EOA qu'en EOO (t(19) $=-6,923 ; \mathrm{p}<0,0001$; calculé sur la racine de l'arc sinus du pourcentage d'énoncés initiés par un verbe).

\begin{tabular}{|c|c|c|c|c|c|}
\hline Noms & Déterminants & Verbes & Pronoms & Autres \\
\hline EOO & $\begin{array}{c}2,06 \% \\
(2,79)\end{array}$ & $\begin{array}{c}18,23 \% \\
(5,18)\end{array}$ & $\begin{array}{c}2,18 \% \\
(3,34)\end{array}$ & $\begin{array}{c}34,82 \% \\
(7,01)\end{array}$ & $\begin{array}{c}42,72 \% \\
(8,24)\end{array}$ \\
\hline EOA & $\begin{array}{c}1,38 \% \\
(1,99)\end{array}$ & $\begin{array}{c}6,36 \% \\
(2,10)\end{array}$ & $\begin{array}{c}8,14 \% \\
(4,13)\end{array}$ & $\begin{array}{c}63,23 \% \\
(5,48)\end{array}$ & $\begin{array}{c}20,89 \% \\
(5,58)\end{array}$ \\
\hline
\end{tabular}

Tableau 8. Pourcentage moyen d'énoncés initiés par un nom, un verbe, un déterminant, un pronom ou un élément d'une autre catégorie en fonction des intentions communicatives (écart-type entre parenthèses).

\section{Conclusion}

Nous observons un effet du contexte communicatif sur le type d'intentions communicatives réalisées par les mères : les énoncés orientés vers les objets sont plus nombreux en lecture d'album alors que ceux 
orientés vers les actions sont plus présents au cours du jeu avec la maison de poupée. Ces résultats vont dans le sens de ceux de Choi (2000) qui observe que les mères coréennes focalisent davantage l'attention de leurs enfants sur les actions en contexte de jeu et davantage sur les objets et les personnes en contexte de lecture d'album. Par contre, notre hypothèse d'une préférence des mères francophones pour les objets et les personnes (vs. les actions) est invalidée puisqu'elles attirent l'attention de leur enfant sur des réalités différentes en fonction de la situation. Ce comportement est différent de celui des mères anglophones qui orientent leurs énoncés sur les noms à la fois dans la lecture d'album sans texte et dans les séances de jeu (Choi, 2000). Ainsi, même si les mères françaises parlent une langue que l'on peut considérer comme orientée vers les noms (noun-friendly), le contexte de production semble jouer un rôle plus important.

Par ailleurs, le contexte communicatif n'influence pas la longueur des énoncés maternels : dans les deux contextes, les énoncés multimots sont les plus nombreux. Par contre, le contexte communicatif joue un rôle sur la composition grammaticale des énoncés monomots : les énoncés monomots qui comportent un verbe se trouvent davantage en contexte de jeu avec la maison de poupée qu'en contexte de lecture d'album. Enfin, le contexte communicatif a une influence sur les éléments qui sont placés en position finale d'énoncés : la proportion de verbes en position finale est plus importante en contexte de maison de poupée que de lecture d'album. Dans tous les cas étudiés, les verbes sont davantage placés en position saillante (seuls, en position finale) dans la situation de jeu que dans la situation de lecture partagée. Ainsi, contrairement à nos attentes, dans la situation de jeu les verbes sont particulièrement mis en valeur par la position qu'on leur fait occuper dans les énoncés alors que les noms occupent des positions identiques dans les deux contextes.

Ce travail a permis également d'observer la réalisation linguistique de deux types d'intentions communicatives: l'intention d'orienter l'attention des enfants vers les personnes et les objets et l'intention d'orienter leur attention sur les actions. Ces deux types d'intentions sont réalisés par le biais d'outils linguistiques et de structures syntaxiques différentes. On note tout d'abord que les énoncés monomots composés d'un verbe uniquement sont plus fréquents dans les énoncés orientés vers les actions que vers les objets. Ensuite, le pourcentage moyen de verbes en position finale et initiale est significativement plus élevé dans les énoncés orientés vers les actions que ceux orientés vers les objets.

Dans ce travail, la préférence des mères françaises pour les noms est remise en question. Il semblerait en effet que les contextes de production jouent un rôle plus essentiel sur le choix des intentions communicatives à exprimer ainsi que sur le choix des outils linguistiques invoqués pour les réaliser que les caractéristiques typologiques et fonctionnelles de la langue employée. Pour asseoir cette idée, nous envisageons des analyses translinguistiques complémentaires sur des langues typologiquement différentes en particulier dans le domaine de la morphosyntaxe des noms et des verbes.

Ce résultat nous oblige également à repenser le lien possible entre input et acquisition. Sans aller jusqu'à le nier complètement, il faudrait peut-être l'envisager de manière moins directe ou encore de manière décalée dans le temps. En outre, d'autres caractéristiques de l'input que nous n'avons pas étudiées ici peuvent jouer un rôle sur la trajectoire acquisitionnelle des enfants. Ainsi, avant de « jeter bébé avec l'eau du bain », des analyses complémentaires sont à mener.

\section{Références bibliographiques}

Amery, H. \&Cartwright, S. (1986). The Farm Picture Book. Macmillan.

Bassano, D. (1998). Sémantique et syntaxe dans l'acquisition des classes de mots : l'exemple des noms et des verbes en français. Langue Française, 118, 26-48.

Bassano, D. (2000). Early development of nouns and verbs in French: exploring the interface between lexicon and grammar. Journal of Child Language, 27, 521-559.

Bassano, D., Maillochon, I. \& Eme, E. (1998). Developmental changes and variability in early lexicon: A study of French children's naturalistic productions. Journal of Child Language, 25, 493-531. 
Bornstein, M. H., Cote, L. R., Maital, S., Painter, K., Park, S., Pascual, L., Pêcheux, M., Ruel, J., Venuti, P. \& Vyt, A. (2004). Cross-Linguistic Analysis of Vocabulary in Young Children: Spanish, Dutch, French, Hebrew, Italien, Korean, and American English. Child Development, 4, 1115-1139.

Caselli, M. C., Bates, E., Casadio, P., Fenson, J., Fenson, L., Sanderl, L. \& Weir, J. (1995). A cross linguistic study of early lexical development. Cognitive Development, 10(2), 159-200.

Cheng, S. W. (1994). Beginning words of three children acquiring Mandarin Chinese. Manuscript, National Chung Cheng University. Taiwan.

Choi, S. (2000). Caregiver input in English and Korean: use of nouns and verbs in book reading and toy play contexts. Journal of Child Language, 27, 69-96.

Choi, S. \& Gopnik, A. (1995). Early acquisition of verbs in Korean: a cross-linguistic study. Journal of Child Language, 22, 497-527.

De Houwer, A. \& Gillis, S. (1998). Dutch child language: an overview. In Gillis, S. \& De Houwer, A. (éd.), The acquisition of Dutch, Amsterdam: John Benjamins, 1-100.

de León, L. (1998). The emergent participant: Patterns of socialization of Tzotzil (Mayan) infants. Journal of Linguistic Anthropology, 8(2), 131-161.

Dromi, E. (1987). Early Lexical Development. Cambridge: C. U. P.

Fernald, A., \& Morikawa, H. (1993). Common themes and cultural variations in Japanese and American mothers' speech to infants. Phonetica, 57, 242-254.

Gentner, D. (1982). Why nouns are learned before verbs: linguistic relativity versus natural partitioning. In Kuczaj, S. A. (éd.), Language Development, Vol. 2: Language, Thought and Culture, Hillsdale, NJ: Erlbaum, 301-334.

Gillis, S., \& Verlinden, A. (1988). Nouns and early verbs in early lexical development: Effects of input frequency? Antwerp Papers of Linguistics, 54. Antwerp: Antwerp University Press.

Goldfield, B. (1993). Noun bias in maternal speech to one-year olds. Journal of Child Language, 20, 85-99.

Gopnik, A., Choi, S. \& Baumberger, T. (1996). Cross-linguistic differences in semantic and cognitive development. Cognitive Development, 11(2), 197-227.

Harris, M. (1990). French. In: Comrie, B. (éd.), The world's major languages, Oxford: Oxford Univ. Press, 210-235.

Jackson-Maldonado, T. D., Marchman, V., Bates, E. \& Gutienez-Clennen, V. (1993). Early lexical development in spanish-speaking infants and toddlers. Journal of Child Language, 20(3), 523-549.

Kern, S., (2007). Lexicon development in French-speaking infants. First Language, 27 (3), 227-250.

Kern, S. \& Gayraud, F. (2010). Inventaire Français du Développement Communicatif. Grenoble : Éditions La Cigale.

Kim, M., McGregor, K. K. \& Thompson, C. K. (2000). Early Lexical Development in English and Korean speaking children: language-general and language-specific patterns. Journal of Child Language, 27, 225-254.

MacWhinney, B. (2000) The CHILDES Project: Tools for Analyzing Talk. Volume 1: Transcription format and programs. Volume 2: The Database. Mahwah, NJ: Lawrence Erlbaum Associates.

Maital, S. L., Dromi, E., Sagi, A. \& Bornstein, M. H. (2000) The Hebrew Communicative Development Inventory: language specific properties and cross-linguistic generalizations. Journal of Child Language, 27, 43-67.

Ogura, T., Dale, P. S., Yamashita, Y., Murase, T. \& Mahieu, A. (2006). The use of nouns and verbs by Japanese children and their caregivers in book-reading and toy-playing contexts. Journal of Child Language, 33, 1-29.

Parisse, C. \& Le Normand, M. T. (2000). How children build their morphosyntax: the case of French. Journal of Child Language, 27(2), 267-292.

Poulin-Dubois, D., Graham, S. \& Sippola, Z. (1995). Early lexical development: the contribution of parental labelling and infants categorization skills abilities. Journal of Child Language, 22, 325-343.

Slobin, D. (1985). Cross linguistic Study of language acquisition. Hillsdale, NJ: Lawrence Erlbaum Associates.

Tardif, T. (1996). Nouns are not always learned before verbs: evidence from Mandarin speakers'early vocabularies. Developmental Psychology, 32, 491-504. 
978-2-7598-0534-1, Paris, 2010, Institut de Linguistique Française

Psycholinguistique et acquisition

DOI $10.1051 / \mathrm{cmlf} / 2010124$

Tardif, T., Gelman, A. S. \& Xu, F. (1999). Putting the Noun Bias in Context: A Comparison of English and Mandarin. Child Development, 70(3), 620-635.

Tardif, T., Shatz, M., \& Naigles, L. (1997). Caregiver speech and children's use of nouns versus verbs: A comparison of English, Italian and Mandarin. Journal of Child Language, 24, 535-565. 